\title{
Interrelationships between Skin Structure, Function, and Microbiome of Pregnant Females and Their Newborns: Study Protocol for a Prospective Cohort Study
}

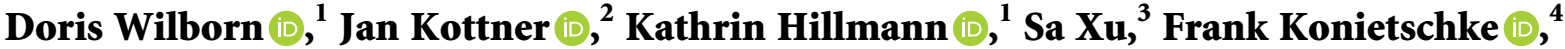 \\ and Ulrike Blume-Peytavi $\mathbb{D}^{1}$ \\ ${ }^{1}$ Clinical Research Center for Hair and Skin Science, Department of Dermatology, Venereology and Allergology, \\ Charité-Universitätsmedizin Berlin, Charitéplatz 1, Berlin 10117, Germany \\ ${ }^{2}$ Institute of Clinical Nursing Science, Charité-Universitätsmedizin Berlin, Charitéplatz 1, Berlin 10117, Germany \\ ${ }^{3}$ Dehaa Rossun Research Center, Kowloon, Hongkong, China \\ ${ }^{4}$ Department of Biometry and Clinical Epidemiology, Charité-Universitätsmedizin Berlin, Charitéplatz 1, Berlin 10117, Germany
}

Correspondence should be addressed to Doris Wilborn; doris.wilborn@charite.de

Received 12 May 2021; Accepted 28 October 2021; Published 17 November 2021

Academic Editor: Craig G. Burkhart

Copyright $\odot 2021$ Doris Wilborn et al. This is an open access article distributed under the Creative Commons Attribution License, which permits unrestricted use, distribution, and reproduction in any medium, provided the original work is properly cited.

\begin{abstract}
Background. Pregnancy leads to several skin changes, but evidence about structural and functional skin changes is scarce. Findings on skin structure and function in children in their first year reveal rapid skin maturation, but evidence indicates that in particular, water holding and transport mechanisms are different from adults. Important questions include whether maternal cutaneous properties predict infant skin condition, and if so, how. This is especially relevant for the skin's microbiome because it closely interacts with the host and is assumed to play a role in many skin diseases. Therefore, the study objective is to explore characteristics of skin and hair of pregnant women and their newborns during pregnancy and in the first six months after delivery and their associations. Methods. The study has an observational longitudinal design. We are recruiting pregnant females between 18 and 45 years using advertisement campaigns in waiting areas of gynecologists and hospital's outpatient services. A final sample size of $n=100$ women is the target. We perform noninvasive, standardized skin, hair, and skin microbiome measurements. We establish the baseline visit during pregnancy until at the latest four weeks before delivery. We schedule follow-up visits four weeks and six months after birth for mothers and their newborns. We will calculate descriptive statistical methods using frequencies and associations over time depending on scale levels of the measurements. Discussion. The majority of previous studies that have investigated infants' skin microbiome and its associations used cross-sectional designs and focused on selected characteristics in small samples. In our longitudinal study, we will characterize a broad range of individual and environmental characteristics of mothers and their newborns to evaluate interrelationships with skin parameters and their changes over time. Considering the combination of these multiple variables and levels will allow for a deeper understanding of the complex interrelationship of the newborn's skin maturation. This trial is registered with ClinicalTrials.gov (Identifier: NCT04759924).
\end{abstract}

\section{Background}

The skin is widely considered the largest organ of the human body, and it fulfills a variety of essential functions. One of the most important functions is protection. However, there are certain periods in life when skin health is challenged [1]. Two of these periods are during pregnancy for women and during the first few months after birth for both mothers and their newborns. It is well known that pregnant women may undergo a wide range of skin and hair changes such as pigmentary, vascular, hair growth, nail, and connective tissue alterations [2-6]. These effects are also assumed to continue after childbirth [7]. The scalp hair is particularly affected: hormonal changes during pregnancy lead to a high anagen rate of nearly $90 \%$ of all hair follicles during the second and third quarters of pregnancy [8]. However, some 
women experience postpartum diffuse hair loss, which is enhanced in women already having hair loss problems prior to pregnancy. This hair growth disorder is known as telogen effluvium, lasting between six weeks and up to six months after delivery [9]. But why there are significant individual differences in postnatal hair loss remains unclear. So detailed evidence about the structure and function of skin and hair changes during and after pregnancy is largely lacking.

In contrast, evidence about skin function and skin structure in newborns and infants is more common. Immediately after birth, the rapid skin barrier maturation is well studied [10], but evidence indicates that in particular, the water holding and transport mechanisms are very different to adults in the first year of life [11]. Transepidermal water loss (TEWL) measurements indicate that after the first month after birth, TEWL seems to become similar to the adult skin $[10,12,13]$. Until one year of age, TEWL increases $[10,14,15]$, and $\mathrm{pH}$ values of the skin surface decline from immediately after birth to several months later, indicating skin barrier maturation $[10,15,16]$.

In recent years, there has been an increasing interest in associations of the skin microbiome and skin health [17]. The above-described physiological changes of the skin from birth during the first year of life may also interact with the skin microbiome [18]. Oranges et al. conclude that the skin microbiome of newborns resembles the microbiome of moist skin sites in adults [13]. Other study results indicate associations between the richness and diversity of the microbiome, the skin, and environmental properties [18-21]. This signifies complex interrelationships between physiological development, microbial colonization and growth, and external influences.

Until now, few studies have explored possible associations of skin function and microbiome between mothers and newborns. Dominguez-Bello et al. showed that the method of delivery (vaginal or via C-section) is linked to the diversity of the cutaneous bacterial colonization [19]. Additionally, results of 50 mother-child pairs indicate similarities of the children's bacterial genera to those of their own mothers [22]. Other study results suggest associations between environmental conditions, age, and the skin microbiome $[23,24]$.

However, most study results are based on cross-sectional study designs, ignoring changes over time. Because of the multiple interactions between skin structure, function, and microbiome, it is necessary to consider and measure all aspects over time.

\section{Methods/Design}

2.1. Aim. The aim is to measure skin and hair characteristics of mothers and newborns over time and determine demographic, environmental, and health characteristics associated with skin microbiome development.

Therefore, we will answer the following research questions:

(1) Are there associations between health, skin, and hair characteristics of mothers and newborns?
(2) How does the skin microbiome change during pregnancy and after birth?

(3) Do maternal health and skin characteristics predict the skin characteristics of newborns and infants?

2.2. Study Design. We perform a longitudinal, descriptive cohort study including females during pregnancy and puerperium, and their newborns until six months of age shown in Table 1.

2.3. Setting. We invite women after the first pregnancy trimester from the federal state of Berlin (Germany) during their visits to gynecologists and/or midwives. We conduct inclusion and all study visits and measurement procedures at the Clinical Research Center for Hair and Skin Science at the Department of Dermatology at the Charité-Universitätsmedizin Berlin (Germany). If they meet the eligibility criteria, women are included in the study. Upon inclusion in the study after the baseline visit, we follow up with the women and their newborns four weeks after delivery and again six months after delivery.

2.4. Participants. Participants are pregnant females between 18 and 45 years old and residing in Berlin (Germany). Prerequisites for study inclusion are individual written informed consent, clinically healthy skin and hair appearance, as well as being free of any dermatological condition. Skin afflictions, which may interfere with the study assessments, especially atopic dermatitis, scars, or other lesions at the investigational site result in exclusion. Women must be of general good and stable health as confirmed by medical history and by a physical examination. They must accept to abstain from sunbathing and solariums and to agree to stay consistent in using their individual skin cleaning and caring routines during the study.

Women are excluded due to regular smoking, regular alcohol intake, or criteria related to diseases such as any dermatological condition or skin affliction, which may interfere with the study assessments. These could be any inflammatory dermatosis (psoriasis, atopic dermatitis, or other lesions at the investigational sites) or clinically significant, possibly unstable medical conditions due to pregnancy such as gestosis, eclampsia, or thrombosis. Criteria related to treatments and products such as current topical or systemic treatment possibly affecting the skin (diuretics, cholesterollowering drugs, and hormones) during the past four weeks are further exclusion criteria. Additionally, therapeutic ultraviolet radiation or increased ultraviolet exposure within six weeks before inclusion leads to exclusion. For safety, other medical reasons, and any individual reasons, study participation can be stopped.

2.5. Variables. Due to the descriptive and exploratory nature of this study, we assess a broad range of characteristics and variables. Skin structure is measured by means of skin surface topography, epidermal thickness, skin stiffness, and skin elasticity. Skin function is measured by means of 
TABle 1: Timeline for women and newborns.

\begin{tabular}{lccc}
\hline & $\begin{array}{c}\text { Inclusion baseline visit during } \\
\text { pregnancy } \\
\left(16^{\text {th }}-36^{\text {th }} \text { week) }\right. \\
\text { t0 }\end{array}$ & $\begin{array}{c}\text { Delivery }+4 \text { weeks } \\
\text { t1 }\end{array}$ & $\begin{array}{c}\text { Delivery+6 months (end } \\
\text { of the study) } \\
\text { t2 }\end{array}$ \\
\hline Informed consent & $x$ & $x$ newborn & $x$ newborn \\
Inclusion/exclusion criteria & $x$ & $x^{1}$ & $x^{1}$ \\
Medical examination & $x$ & $x^{1}$ & $x^{1}$ \\
Dermatological examination & $x$ & $x^{1}$ & $x^{1}$ \\
Skin measurements & $x$ & $x^{1}$ & $x^{1}$ \\
Skin microbiome & $x$ & $x^{1}$ & $x^{1}$ \\
Hair metrix analysis & $x$ & & \\
\hline
\end{tabular}

$x^{1}=$ visit for mother and baby.

stratum corneum hydration (SCH), TEWL, and skin surface $\mathrm{pH}$, which are established parameters to characterize the skin barrier function. Skin microbiome is defined as bacterial diversity, and in this study, the relative abundance of phylotypes, (operational taxonomic units, OTUs) will be measured. Hair growth is measured on the central and occipital scalp by means of hair density and hair widths. The definitions of the variables are shown in Table 2 and further explained in the following paragraph.

2.6. Data Sources/Measurement. We train all data collectors in accurately obtaining the variables of interest. We use paper source data and electronic case report forms to document all study variables of interest.

2.6.1. Dermatological Examination. The investigator performs the dermatological examination during the visits together with a subject's interview on medical history and treatments to check the subject's eligibility.

2.6.2. Measurements of Skin Physiology. We measure the physiological skin parameters of the females and the newborns at one area of the right inner forearm. All measurement procedures are based on internal standardized operating procedures as part of the quality management system of the study site. Before the assessment measurements, subjects rest for 30 minutes in a room with controlled environmental conditions $\left(22^{\circ} \mathrm{C} \pm 2^{\circ} \mathrm{C}\right.$ room temperature; $50 \% \pm 10 \%$ relative humidity) for acclimatization. During acclimatization, the skin of the investigational sites is uncovered and exposed to ambient air. The parameters are measured in the following order: skin swab, TEWL, SCH, $\mathrm{pH}$, Cutometry, OCT, and Visioscan. The order and exact skin areas are arranged in such a way that any measurement procedure will not affect the readings of the subsequent measurement [25].

2.6.3. Skin Surface Topography. Based on the definition of the EEMCO guidance [26], the skin surface topography is measured with "Visioscan VC 98 USB" (Courage + Khazaka Electronics GmbH, Germany). The skin is scanned with a high-resolution camera to measure mean roughness. Mean roughness $(\mathrm{Rz})$ is presented in means $(\mathrm{SD})$ and median (IQR).
2.6.4. Epidermal Thickness. Optical coherence tomography (OCT; THORLABS TELESTO, Spectral Domain OCT System) allows for a noninvasive imaging of the epidermis $[27,28]$. Based on the obtained images, epidermal thickness is calculated using internal standardized operational procedures.

2.6.5. Skin Stiffness and Elasticity. The total extensibility of the skin (Uf, $\mathrm{mm}$ ) is measured using the "Cutometer MPA 580" (Courage + Khazaka Electronics GmbH, Germany) according to guidance by the EECMO group for the assessment of biomechanical skin properties [29].

2.6.6. Transepidermal Water Loss. TEWL ( $g$ per hour per $\mathrm{m}^{2}$ ) is measured using the "Tewameter TM 300" (Courage + Khazaka, Electronics GmbH, Germany). This is an open chamber method, and the results are compared to published reference values in adults and newborns [10,30]. Empirical evidence supports the reliability of this estimate at the volar forearm [31].

2.6.7. Skin Surface $p H$. Skin $p H$ is measured using the "Skinph-Meter ${ }^{\circledR} \mathrm{PH} 905$ device" (Courage + Khazaka, Electronics $\mathrm{GmbH}$, Germany). This investigation is highly reliable at the volar forearm [31] when applying the standard operating procedures. Reference values of human skin have been reported to range from 4 to $6[32,33]$.

2.6.8. Stratum Corneum Hydration. SCH is measured by using the "Corneometer CM 825" (Courage + Khazaka, Electronics $\mathrm{GmbH}$, Germany). The measurement is based on the differences in the dielectric constant of water and other substances within the stratum corneum. The arbitrary units for $\mathrm{SCH}$ measurements range from 0 to 120 , with higher readings indicating higher $\mathrm{SCH}[34]$.

2.6.9. Skin Microbiome and Analysis. We follow the analytic test methods put forward by McInnes and Cutting in the "Human microbiome project," for describing the microbiome distribution of bacterial species in this study [35]. According to the latest recommendations by the involved test laboratory, a swab is taken with aseptic techniques at the volar forearm. The swab is moistened with sterile $\mathrm{NaCl}$ 
TABle 2: Variables.

\begin{tabular}{|c|c|c|c|c|c|}
\hline Variables & $\begin{array}{c}\text { Statistics/ } \\
\text { level of scales }\end{array}$ & $\begin{array}{l}\text { Possible range of } \\
\text { measurements }\end{array}$ & $\begin{array}{l}\text { Time of } \\
\text { measurement } \\
\text { Women }\end{array}$ & $\begin{array}{c}\text { Time of } \\
\text { measurement } \\
\text { Newborn/infant }\end{array}$ & $\begin{array}{l}\text { Source of } \\
\text { information }\end{array}$ \\
\hline Age & $\begin{array}{c}\text { Metric } \\
\text { variable }\end{array}$ & $\begin{array}{l}\text { In years, women } \\
\text { In days/months, } \\
\text { newborn/infant } \\
18-45 \text { years, women } \\
0-28 \text { d, newborn } \\
1-6 \text { months, infant }\end{array}$ & Baseline, t0 & $\begin{array}{l}\text { Delivery }+28 \mathrm{~d}, \mathrm{t} 1 \\
\text { Delivery }+6 \text { months, } \\
\mathrm{t} 2\end{array}$ & $\begin{array}{c}\text { ID card, woman } \\
\text { "U-heft," newborn/ } \\
\text { infant } \\
\text { U3: } 4^{\text {th }}-5^{\text {th }} \text { week } \\
\text { U5: } 6^{\text {th }}-7^{\text {th }} \text { month }\end{array}$ \\
\hline Weight & $\begin{array}{c}\text { Metric } \\
\text { variable }\end{array}$ & $\begin{array}{c}\text { In } \mathrm{kg} \text {, woman } \\
\text { In } \mathrm{kg} \text {, newborn/infant }\end{array}$ & $\begin{array}{c}\text { Baseline, } \mathrm{t} 0 \\
\text { Delivery }+28 \mathrm{~d}, \mathrm{t} 1 \\
\text { Delivery }+6 \text { months, } \\
\mathrm{t} 2\end{array}$ & $\begin{array}{l}\text { Delivery }+28 \mathrm{~d}, \mathrm{t} 1 \\
\text { Delivery }+6 \text { months, } \\
\mathrm{t} 2\end{array}$ & $\begin{array}{c}\text { Measurement } \\
\text { "U-heft," newborn/ } \\
\text { infant } \\
\text { U3: } 4^{\text {th }}-5^{\text {th }} \text { week } \\
\text { U5: } 6^{\text {th }}-7^{\text {th }} \text { month } \\
\text { Self-reporting, }\end{array}$ \\
\hline Height & $\begin{array}{c}\text { Metric } \\
\text { variable }\end{array}$ & $\begin{array}{l}\text { In } \mathrm{cm} \text {, woman } \\
\text { In } \mathrm{cm} \text {, newborn/ } \\
\text { infant }\end{array}$ & Baseline, t0 & $\begin{array}{l}\text { Delivery }+28 \mathrm{~d}, \mathrm{t} 1 \\
\text { Delivery }+6 \text { months, } \\
\mathrm{t} 2\end{array}$ & $\begin{array}{l}\text { woman } \\
\text { "U-heft," newborn/ } \\
\text { infant } \\
\text { U3: } 4^{\text {th }}-5^{\text {th }} \text { week } \\
\text { U5: } 6^{\text {th }}-7^{\text {th }} \text { month }\end{array}$ \\
\hline Sex & $\begin{array}{l}\text { Nominal } \\
\text { variable }\end{array}$ & $\begin{array}{l}\text { Female } \\
\text { Male }\end{array}$ & & Delivery $+28 \mathrm{~d}, \mathrm{t} 1$ & $\begin{array}{c}\text { "U-heft," newborn/ } \\
\text { infant } \\
\text { U1 delivery }\end{array}$ \\
\hline Habitation & $\begin{array}{l}\text { Nominal } \\
\text { variable }\end{array}$ & $\begin{array}{l}\text { Urban } \\
\text { Rural }\end{array}$ & $\begin{array}{c}\text { Baseline, } \mathrm{t} 0 \\
\text { Delivery }+28 \mathrm{~d}, \mathrm{t} 1 \\
\text { Delivery }+6 \text { months, } \\
\mathrm{t} 2\end{array}$ & $\begin{array}{l}\text { Delivery }+28 \mathrm{~d}, \mathrm{t} 1 \\
\text { Delivery }+6 \text { months, } \\
\mathrm{t} 2\end{array}$ & $\begin{array}{l}\text { Self-reporting, } \\
\text { woman }\end{array}$ \\
\hline $\begin{array}{l}\text { Newborn/infant's } \\
\text { environment }\end{array}$ & $\begin{array}{l}\text { Nominal } \\
\text { variable }\end{array}$ & $\begin{array}{l}\text { Apartment } \\
\text { House }\end{array}$ & & $\begin{array}{c}\text { Delivery }+28 \mathrm{~d}, \mathrm{t} 1 \\
\text { Delivery }+6 \text { months, } \\
\mathrm{t} 2\end{array}$ & $\begin{array}{l}\text { Self-reporting, } \\
\text { woman }\end{array}$ \\
\hline Course of delivery & $\begin{array}{l}\text { Nominal } \\
\text { variable }\end{array}$ & $\begin{array}{l}\text { Vaginal birth } \\
\text { Caesarean }\end{array}$ & Delivery $+28 \mathrm{~d}, \mathrm{t} 1$ & & $\begin{array}{c}\text { "U-heft," newborn/ } \\
\text { infant } \\
\text { U1 delivery }\end{array}$ \\
\hline Vaginal seeding & $\begin{array}{l}\text { Nominal } \\
\text { variable }\end{array}$ & $\begin{array}{l}\text { Yes } \\
\text { No }\end{array}$ & & Delivery $+28 \mathrm{~d}, \mathrm{t} 1$ & $\begin{array}{l}\text { Self-reporting, } \\
\text { woman }\end{array}$ \\
\hline Newborn/infant nutrition & $\begin{array}{l}\text { Nominal } \\
\text { variable }\end{array}$ & $\begin{array}{c}\text { Breastfeeding } \\
\text { Cow-milk-based } \\
\text { infant formula } \\
\text { (including } \\
\text { hypoallergenic (HA) } \\
\text { based formula) } \\
\text { feeding or } \\
\text { combination }\end{array}$ & & $\begin{array}{l}\text { Delivery }+28 \mathrm{~d}, \mathrm{t} 1 \\
\text { Delivery }+6 \text { months, } \\
\mathrm{t} 2\end{array}$ & $\begin{array}{l}\text { Self-reporting, } \\
\text { woman }\end{array}$ \\
\hline $\begin{array}{l}\text { Period of newborn/infant } \\
\text { nutrition (supplementary } \\
\text { feeding) }\end{array}$ & $\begin{array}{c}\text { Metric } \\
\text { variable }\end{array}$ & $\begin{array}{l}\text { From week } x \text { to week } \\
\qquad \mathrm{x}\end{array}$ & & $\begin{array}{c}\text { Delivery }+28 \mathrm{~d}, \mathrm{t} 1 \\
\text { Delivery }+6 \text { months, } \\
\mathrm{t} 2\end{array}$ & $\begin{array}{l}\text { Self-reporting, } \\
\text { woman }\end{array}$ \\
\hline Gestational age at birth & $\begin{array}{c}\text { Metric } \\
\text { variable }\end{array}$ & In weeks & & Delivery $+28 \mathrm{~d}, \mathrm{t} 1$ & $\begin{array}{c}\text { "U-heft," newborn/ } \\
\text { infant } \\
\text { U1 delivery }\end{array}$ \\
\hline $\begin{array}{l}\text { Dermatological skin } \\
\text { assessment, mother }\end{array}$ & $\begin{array}{l}\text { Nominal } \\
\text { variable }\end{array}$ & Coded by ICD11 & $\begin{array}{c}\text { Baseline, } \mathrm{t} 0 \\
\text { Delivery }+28 \mathrm{~d}, \mathrm{t} 1 \\
\text { Delivery }+6 \text { months, } \\
\mathrm{t} 2\end{array}$ & & Study MD \\
\hline
\end{tabular}


TABle 2: Continued.

\begin{tabular}{|c|c|c|c|c|c|}
\hline Variables & $\begin{array}{l}\text { Statistics/ } \\
\text { level of scales }\end{array}$ & $\begin{array}{l}\text { Possible range of } \\
\text { measurements }\end{array}$ & $\begin{array}{c}\text { Time of } \\
\text { measurement } \\
\text { Women } \\
\end{array}$ & $\begin{array}{c}\text { Time of } \\
\text { measurement } \\
\text { Newborn/infant }\end{array}$ & $\begin{array}{l}\text { Source of } \\
\text { information }\end{array}$ \\
\hline $\begin{array}{l}\text { Dermatological skin } \\
\text { assessment, newborn/infant }\end{array}$ & $\begin{array}{l} \\
\text { (i) Eczema } \\
\text { (ii) Dry skin } \\
\text { (iii) Itchy } \\
\text { skin } \\
\text { (iv) Rash } \\
\text { (v) Flexural } \\
\text { dermatitis } \\
\text { and/or visible } \\
\text { dermatitis } \\
\text { (vi) Diaper } \\
\text { dermatitis } \\
\text { (vii) Hand, } \\
\text { mouth, and } \\
\text { foot disease }\end{array}$ & $\begin{array}{l}\text { EASI score: } \\
\text { (i) Erythema } \\
\text { (ii) Edema/papulation } \\
\text { (iii) Excoriation } \\
\text { (iv) Lichenification } \\
\text { EASI score: } \\
\text { (i) Severity of signs } \\
\text { (1) 0-3 } \\
\text { (2) None severe } \\
\text { Dry skin: } \\
\text { (ii) Desquamation } \\
\text { fissures/rhagades } \\
\text { (iii) Erythema } \\
\text { (iv) Pruritus } \\
\text { Itchy skin: } \\
\text { (i) If yes: continuous/ } \\
\text { intermittent } \\
\text { Rash: yes/no } \\
\text { Diaper dermatitis: } \\
\text { yes/no } \\
\text { Hand, mouth, and } \\
\text { foot disease: yes/no }\end{array}$ & & $\begin{array}{c}\text { Delivery }+28 \mathrm{~d}, \mathrm{t} 1 \\
\text { Delivery }+6 \text { months, } \\
\mathrm{t} 2\end{array}$ & Study MD \\
\hline Skin care, mother & $\begin{array}{l}\text { Open-ended } \\
\text { question }\end{array}$ & (2) & $\begin{array}{c}\text { Baseline, } \mathrm{t} 0 \\
\text { Delivery }+28 \mathrm{~d}, \mathrm{t} 1 \\
\text { Delivery }+6 \text { months, } \\
\mathrm{t} 2\end{array}$ & & $\begin{array}{l}\text { Self-reporting, } \\
\text { woman }\end{array}$ \\
\hline Skin care, newborn/infant & $\begin{array}{l}\text { Open-ended } \\
\text { question }\end{array}$ & & & $\begin{array}{c}\text { Delivery }+28 \mathrm{~d}, \mathrm{t} 1 \\
\text { Delivery }+6 \text { months, } \\
\mathrm{t} 2\end{array}$ & $\begin{array}{l}\text { Self-reporting, } \\
\text { woman }\end{array}$ \\
\hline Care givers at home & $\begin{array}{l}\text { Nominal } \\
\text { variable }\end{array}$ & $\begin{array}{l}\text { (i) Father } \\
\text { (ii) Sibling } \\
\text { (iii) Grandparents } \\
\text { (iv) Au pair } \\
\text { (v) Nursery nurse } \\
\text { (vi) Other }\end{array}$ & & $\begin{array}{c}\text { Delivery }+28 \mathrm{~d}, \mathrm{t} 1 \\
\text { Delivery }+6 \text { months, } \\
\mathrm{t} 2\end{array}$ & $\begin{array}{l}\text { Self-reporting, } \\
\text { woman }\end{array}$ \\
\hline Furred pets at home & $\begin{array}{l}\text { Nominal } \\
\text { variable }\end{array}$ & $\begin{array}{l}\text { (i) Cat } \\
\text { (ii) Dog } \\
\text { (iii) Others }\end{array}$ & $\begin{array}{c}\text { Baseline, } \mathrm{t} 0 \\
\text { Delivery }+28 \mathrm{~d}, \mathrm{t} 1 \\
\text { Delivery }+6 \text { months, } \\
\mathrm{t} 2\end{array}$ & & $\begin{array}{l}\text { Self-reporting, } \\
\text { woman }\end{array}$ \\
\hline $\begin{array}{l}\text { The atopic predisposition of } \\
\text { the mother (at least one } \\
\text { parent or sibling with } \\
\text { physician-diagnosed AD, } \\
\text { asthma, or allergic rhinitis/ } \\
\text { rhinoconjunctivitis as } \\
\text { reported by at least one } \\
\text { parent but in otherwise } \\
\text { good overall health) }\end{array}$ & $\begin{array}{l}\text { Nominal } \\
\text { variable }\end{array}$ & Yes/no & Baseline, t0 & & $\begin{array}{l}\text { Self-reporting, } \\
\text { woman }\end{array}$ \\
\hline Antibiotic intake & $\begin{array}{l}\text { Nominal } \\
\text { variable }\end{array}$ & Yes/no & $\begin{array}{c}\text { Baseline, } \mathrm{t} 0 \\
\text { Delivery }+28 \mathrm{~d}, \mathrm{t} 1 \\
\text { Delivery }+6 \text { months, } \\
\mathrm{t} 2\end{array}$ & & $\begin{array}{l}\text { Self-reporting, } \\
\text { woman }\end{array}$ \\
\hline Alcohol consumption & $\begin{array}{l}\text { Nominal } \\
\text { variable }\end{array}$ & Yes/no & $\begin{array}{c}\text { Baseline, } \mathrm{t} 0 \\
\text { Delivery }+28 \mathrm{~d}, \mathrm{t} 1 \\
\text { Delivery }+6 \text { months, } \\
\mathrm{t} 2\end{array}$ & & $\begin{array}{l}\text { Self-reporting, } \\
\text { woman }\end{array}$ \\
\hline
\end{tabular}


TABle 2: Continued.

\begin{tabular}{ccccc}
\hline Variables & $\begin{array}{c}\text { Statistics/ } \\
\text { level of scales }\end{array}$ & $\begin{array}{c}\text { Possible range of } \\
\text { measurements }\end{array}$ & $\begin{array}{c}\text { Time of } \\
\text { measurement } \\
\text { Women }\end{array}$ & $\begin{array}{c}\text { Time of } \\
\text { measurement } \\
\text { Newborn/infant }\end{array}$ \\
\hline
\end{tabular}

Skin surface topography roughness:

(i) RA (arithmetic mean roughness) in $\mu \mathrm{m}$

(ii) RZ (arithmetic mean roughness from five sampling length) in $\mu \mathrm{m}$

$\begin{array}{cc} & \text { Metric scaled } \\ \text { variable } \\ \text { Epidermal thickness in } \mu \mathrm{m} \quad \text { Mean (SD) } \\ \text { Median } \\ \text { (IQR) }\end{array}$

Skin stiffness and elasticity total extensibility (Uf, mm)

Transepidermal water loss (TEWL)

Transepidermal water loss through the stratum corneum in $g$ per hour per $\mathrm{m}^{2}$

RA: $0.0-100.0 \mu \mathrm{m}$

RZ: $0.0-100.0 \mu \mathrm{m}$

(IQR)

Skin surface $\mathrm{pH}$

Stratum corneum hydration (SCH)

Skin microbiome Bacterial diversity Relative abundance of phylotypes (operational taxonomic units, OTUs) Top 5 genera
Metric scaled variable

\section{Mean (SD) \\ Median}

(IQR)

$$
\begin{gathered}
0.0-100.0 \mu \mathrm{m} \\
(40-60 \mu \mathrm{m})
\end{gathered}
$$

Metric scaled variable

Mean (SD)

Median

(IQR)

Metric scaled variable

Baseline, $\mathrm{t} 0$

Delivery $+28 \mathrm{~d}$, $\mathrm{t} 1$

Delivery +6 months, $\mathrm{t} 2$

Baseline, $\mathrm{t} 0$

$0.00-0.50$

Uf in $\mathrm{mm}$ /degree

Delivery $+28 \mathrm{~d}, \mathrm{t} 1$

Delivery +6 months,

t2

$0.0-60.0 \mathrm{~g} / \mathrm{m}^{2} / \mathrm{h}$

Delivery $+28 \mathrm{~d}, \mathrm{t} 1$

Delivery +6 months,

$>4$ or $<7.5$

Median

(IQR)

Metric scaled 0-120, arbitrary units, variable

Mean (SD)

Median

(IQR)

whereas higher

readings indicate

higher stratum

corneum hydration

$\mathrm{N}$ number of

Metric scaled variable

Alpha diversity

Shannon

index
Mean (SD) taxonomic groups, for example,

(i) Proteobacteria and Bacteroidetes

(ii) Firmicutes

(iii) Actinobacteria

$N$ amount of bacteria (bacterial load)
Baseline, $\mathrm{t} 0$

Standardized images via visioscan VC 98 USB

(i) RA (arithmetic mean roughness) in $\mu \mathrm{m}$

Delivery $+28 \mathrm{~d}, \mathrm{t} 1$ (ii) RZ (arithmetic mean roughness from five sampling length) in $\mu \mathrm{m}$ Skin area: inner forearm Standardized images via optical coherence Delivery $+28 \mathrm{~d}$, t1 tomography (OCT) Delivery +6 months, Thorlabs' Telesto t2

Epidermal thickness in $\mathrm{mm}$

Skin area: inner forearm

Cutometer MPA 580

total extensibility

Delivery $+28 \mathrm{~d}, \mathrm{t} 1$ Delivery +6 months,

(Uf, mm), structural $\mathrm{t} 2$

elasticity (Ur/Uf)

Skin area: inner forearm

Tewameter 300

Transepidermal

Delivery $+28 \mathrm{~d}, \mathrm{t} 1 \quad$ water loss through Delivery +6 months, the stratum corneum $\mathrm{t} 2$

Baseline, $\mathrm{t} 0$

Delivery $+28 \mathrm{~d}, \mathrm{t} 1$

Delivery +6 months, $\mathrm{t} 2$

Baseline, $\mathrm{t} 0$

Delivery $+28 \mathrm{~d}, \mathrm{t} 1$

Delivery +6 months, t2

Delivery $+28 \mathrm{~d}, \mathrm{t} 1$ Delivery +6 months, t2

Baseline, $\mathrm{t} 0$

Delivery $+28 \mathrm{~d}, \mathrm{t} 1$

Delivery +6 months, $\mathrm{t} 2$

Delivery $+28 \mathrm{~d}$, $\mathrm{t} 1$ Delivery +6 months, $\mathrm{t} 2$ t2

in $g$ per hour per $\mathrm{m}^{2}$

Skin area: inner forearm

Skin pH-meter ${ }^{\circledR} \mathrm{PH}$ 905

Delivery $+28 \mathrm{~d}, \mathrm{t} 1$ Delivery +6 months, t2

Concentration of

hydrogen ions
Skin area: inner forearm

Corneometer CM 825

Capacitance

measurement of hydration

Arbitrary CM units 0-120

Skin area: inner forearm

Skin microbiome Alpha diversity Skin area: inner forearm 
TABLE 2: Continued.

\begin{tabular}{|c|c|c|c|c|c|}
\hline Variables & $\begin{array}{c}\text { Statistics/ } \\
\text { level of scales }\end{array}$ & $\begin{array}{l}\text { Possible range of } \\
\text { measurements }\end{array}$ & $\begin{array}{c}\text { Time of } \\
\text { measurement } \\
\text { Women }\end{array}$ & $\begin{array}{c}\text { Time of } \\
\text { measurement } \\
\text { Newborn/infant }\end{array}$ & $\begin{array}{l}\text { Source of } \\
\text { information }\end{array}$ \\
\hline Hair measurement & $\begin{array}{c}\text { Metric } \\
\text { variable }\end{array}$ & $\begin{array}{c}\text { Hair thickness } \\
\text { Hair density } \\
\text { Vellus-terminal-hair } \\
\text { ratio }\end{array}$ & $\begin{array}{c}\text { Baseline, } \mathrm{t} 0 \\
\text { Delivery }+28 \mathrm{~d}, \mathrm{t} 1 \\
\text { Delivery }+6 \text { months, } \\
\mathrm{t} 2\end{array}$ & $\begin{array}{c}\text { Delivery }+28 \mathrm{~d}, \mathrm{t} 1 \\
\text { Delivery }+6 \text { months, } \\
\mathrm{t} 2\end{array}$ & $\begin{array}{c}\text { Canfield } \\
\text { Hair Metrix average } \\
\text { hair width in } \mu \mathrm{m} \\
\text { Hair count per } \mathrm{cm}^{2} \\
\text { Central scalp area, } \\
\text { occipital hair area } \\
\text { (woman) } \\
\text { central hair area } \\
\text { (newborn) }\end{array}$ \\
\hline Hair colour & $\begin{array}{l}\text { Ordinal } \\
\text { variable }\end{array}$ & $\begin{array}{l}\text { Hair colour scale, } \\
\qquad 1-10\end{array}$ & $\begin{array}{c}\text { Baseline, } \mathrm{t} 0 \\
\text { Delivery }+28 \mathrm{~d}, \mathrm{t} 1 \\
\text { Delivery }+6 \text { months, } \\
\mathrm{t} 2\end{array}$ & $\begin{array}{c}\text { Delivery }+28 \mathrm{~d} \text {, } \mathrm{t} 1 \\
\text { Delivery }+6 \text { months, } \\
\mathrm{t} 2\end{array}$ & $\begin{array}{l}\text { Comparison with } \\
\text { hair colour scale }\end{array}$ \\
\hline
\end{tabular}

$0.9 \%+0.5 \%$ Tween 20 solution and then rubbed back and forth for 30 seconds. Afterwards, the swab head is inserted into the tube containing DNA/RNA Shield of Zymo Research, Irvine, USA. The head of the swab is then aseptically cut from the handle, and the tube cap is screwed back in place. The skin microbiome is defined as bacterial diversity. The relative abundance of phylotypes (operational taxonomic units, OTUs) is measured by the alpha diversity and Shannon index.

2.6.10. Hair Measurements. Hair measurements are taken on the central and occipital head area in women and on the central head area in newborns/infants. Standardized macro photographs are taken to measure hair growth and structure. We use the Visiomed D200 evo camera of Vectra H1 Hair Metrix, Canfield Scientific, Fairfield, USA. The camera is placed on the selected area of the participant's scalp to take a picture as a noninvasive method. At the selected area (central and occipital), the hair is parted with a comb, preparing the hair region for investigation. The following quantitative hair measurements are calculated automatically by the software system: hair count per $\mathrm{cm}^{2}$, sum of hair width per $\mathrm{cm}^{2}$, terminal to vellus ratio, and average hair width $(\mu \mathrm{m})$. Additionally, the hair colour is estimated by a 10-point hair colour scale [36].

\subsection{Bias}

2.7.1. Information Bias. During the study period, the risk of losing participants is addressed with proper information and close support in the beginning and throughout the study. An expense allowance is provided. If a woman is included during the early phase of pregnancy, a phone call is scheduled three months after inclusion or at the latest one week before the due date. This phone call allows us to check the current situation, as well as to check the date for the second visit ( $\mathrm{t} 1)$. Similar to this procedure, the women are called one week before the scheduled third visit ( $t 2)$. If the women are not reachable, phone calls are repeated twice, and in addition, emails are sent and repeated twice. If there is no response, a letter by registered post is sent. If there is no response after all contact attempts, the woman is then excluded from the study.

In order to estimate the outcomes accurately, all investigations are following internal standardized operating or technical procedures, with staff exclusively allocated to the study performing them.

2.7.2. Selection Bias. Due to the sampling of eligible women restricted to one geographical area, a potential sampling bias is addressed by comparing all relevant characteristics of the included women with national data. Due to the voluntary nature of inclusion in the study, a sampling bias might occur. Therefore, relevant characteristics of the included women are compared to the study population to evaluate potential differences. To address a potential response bias, the age distribution and the number of pregnancies of the included women are considered and compared to national data of the mean age of women at delivery of their first child.

2.8. Study Size. Due to the exploratory character of this study, formal sample size calculation is not performed. We aim to include 100 pregnant females and their newborns/ infants to obtain means and proportions with an acceptable range of uncertainty. A loss-to-follow-up of one-third is assumed resulting in a total of $n=150$ mothers to be included.

2.9. Statistical Methods. We will describe the demographic characteristics of the subjects, using absolute and relative frequencies, means, and standard deviations (SD). Metric outcomes will be described using means, SD, medians, and interquartile ranges (IQR) per group and time point. Ordinally, scaled outcomes will be described using medians and IQR per group and time point. Associations of skin parameters between mothers and their newborns/infants will be described using correlation coefficients (Kendall's Tau). Linear mixed models (metric analysis), as well as nonparametric rank-based methods for longitudinal data 
(ordinal data or skewed endpoints), will be used to assess differences between groups over time (and possible interactions). Furthermore, the Munzel test $r$ (nonparametric) or paired $t$ tests (metric observations) will be used to compare paired data. Confidence intervals (95\%) will be computed for the effects of interest. Due to the explanatory character of the study, all statistical analyses are exploratory, and thus, all $P$-values and confidence intervals are considered descriptive. If missing values occur, we discuss them on an individual level and use all available cases in the analysis. All statistical methods will be performed using validated software.

\subsubsection{Methods to Examine Subgroups and Interactions.} The following subgroup analyses are planned: according to age groups of the females, comparisons will be made for Q1/ Q4-age-related groups. Due to the predisposition for atopic dermatitis $(\mathrm{AD})$, an analysis for existing or non-existing $\mathrm{AD}$ will be made. Because of a possible association between mode of delivery and the skin microbiome, comparisons will be made for vaginal and cesarean delivery.

2.9.2. Missing Data Management. Because of the long individual follow-up, the attrition rates are difficult to predict. After the inclusion of $n=150$ women, we assume the number of dropouts to be $n=50$. Because this is a single cohort of women and their newborns, missing data will not be dependent on different exposures. However, we will compare the groups of subjects with missing data and groups of subjects with complete data regarding main outcome variables to estimate potential bias. Secondly, we will estimate the influence of main predictor variables, such as age, on the occurrence of missing data. Based on the proportion of missing values and the assumed bias, appropriate multiple imputation methods for categorical and continuous variables will be applied [37].

2.10. Dissemination Policy. Subjects or parents/guardians have the right to ask the investigator for a summary of the study results, as soon as they are available. Results are planned to be published in an international peer-reviewed scientific journal. The content and structure of the manuscript will follow the STROBE statement for observational studies $[38,39]$. Authorship will follow the criteria of the International Committee of Medical Journal Editors.

\section{Discussion}

Most of the previous studies investigating the infant's skin microbiome and its associations were conducted using cross-sectional designs and focused on selected characteristics in small samples. In our longitudinal study, we are characterizing a broad range of individual and environmental characteristics of mothers and their newborns to evaluate interrelationships with skin parameters and their changes over the period of at least eight months. Because it is well known that cutaneous changes occur rapidly during and after pregnancy and after birth, the chosen times for measurements are likely to capture these changes. Connecting demographic, environmental characteristics, as well as biophysical skin parameters and the skin microbiome has never been done in this setting to date. Considering these multiple variables and levels together will allow for a deeper understanding of the complex interrelationship of the newborn's skin maturation and allow generating hypotheses to be tested in larger confirmatory studies. By analyzing factors influencing the cutaneous microbiome of the newborns, the results can support recommendations for environmental or individual living conditions and may help predict cutaneous risk in newborns and infants based on their mothers.

\section{Trial Status}

The study is based on the study protocol, from 21 October 2020, Version No. 2. The recruitment phase started on 10 March 2021. The approximate month when recruitment will be completed is estimated to be June 2022 .

\section{Abbreviations}

TEWL: Transepidermal water loss

pH: $\quad$ Pondus hydrogenii

SCH: $\quad$ Stratum corneum hydration

EEMCO: Expert group on efficacy measurement of cosmetics and other topical products

OCT: Optical coherence tomography

DNA: Deoxyribonucleic acid

RNA: Ribonucleic acid

SD: $\quad$ Standard deviation

IQR: Interquartile range

AD: $\quad$ Atopic dermatitis

ICH: International conference on harmonization of technical requirements for registration of pharmaceuticals for human use

ICF: Informed consent form

STROBE: Strengthening the reporting of observational studies in epidemiology

\section{Data Availability}

The data used to support the findings of the study are available from the corresponding author upon request.

\section{Ethical Approval}

The Ethics Committee of the Charité-Universitätsmedizin Berlin reviewed the synopsis, the informed consent form (ICF), and the study protocol for the application of the ethical approval. Approval has been granted on the $14^{\text {th }}$ of December 2020. The committee's reference number is EA2/ $184 / 20$.

\section{Conflicts of Interest}

$\mathrm{Sa} \mathrm{Xu}$ is a member of the DehaaRossun Research Center, Hong Kong, and is employed at Lunaler, Hong Kong. Ulrike 
Blume-Peytavi is a scientific member of the Board of the DehaaRossun Research Center.

\section{Authors' Contributions}

UBP, chief investigator, and JK conceived the study; JK led the proposal and protocol development. DW wrote the study protocol. SX and $\mathrm{KH}$ contributed to the development of the study protocol. FK is the trial methodologist. All the authors read and approved the final manuscript.

\section{Acknowledgments}

This observational study is an investigator-initiated trial conducted at the Charite-Universitätsmedizin Berlin, Germany. The project was financially supported by the DehaaRossun Research Center, Kowloon, Hong Kong, by an unrestricted educational grant. This funding organization was included in the development of the study concept but will not be included in the analysis, interpretation, and publication of the results of the study.

\section{References}

[1] C. Surber, N. Dragicevic, and J. Kottner, "Skin care products for healthy and diseased skin," $p H$ of the Skin: Issues and Challenges, vol. 54, pp. 183-200, 2018.

[2] M. M. Muallem and N. G. Rubeiz, "Physiological and biological skin changes in pregnancy," Clinics in Dermatology, vol. 24, no. 2, pp. 80-83, 2006.

[3] R. Vora, R. Gupta, M. Mehta, A. Chaudhari, A. Pilani, and N. Patel, "Pregnancy and skin," Journal of Family Medicine and Primary Care, vol. 3, no. 4, pp. 318-324, 2014.

[4] V. V. Panicker, N. Riyaz, and P. K. Balachandran, "A clinical study of cutaneous changes in pregnancy," Journal of epidemiology and global health, vol. 7, no. 1, pp. 63-70, 2017.

[5] P. Ciechanowicz, M. Sikora, K. Taradaj et al., "Skin changes during pregnancy. Is that an important issue for pregnant women?” Ginekologia Polska, vol. 89, no. 8, pp. 449-452, 2018.

[6] G. M. Martins-Costa and R. Bakos, "Total body photography and sequential digital dermoscopy in pregnant women," Dermatology Practical and Conceptual, vol. 9, no. 2, pp. 126-131, 2019.

[7] G. Boyer, N. Lachmann, G. Bellemère, C. De Belilovsky, and C. Baudouin, "Effects of pregnancy on skin properties: a biomechanical approach," Skin Research and Technology, vol. 24, no. 4, pp. 551-556, 2018.

[8] C. Piérard-Franchimont and G. E. Piérard, "Alterations in hair follicle dynamics in women," BioMed Research International, vol. 2013, Article ID 957432, , 2013.

[9] E. Thom, "Pregnancy and the hair growth cycle: anagen induction against hair growth disruption using Nourkrin $\left({ }^{\circledR}\right)$ with $\operatorname{Marilex}\left({ }^{\circledR}\right)$, a proteoglycan replacement therapy," Journal of Cosmetic Dermatology, vol. 16, no. 3, pp. 421-427, 2017.

[10] L. Ludriksone, N. Garcia Bartels, V. Kanti, U. Blume-Peytavi, and J. Kottner, "Skin barrier function in infancy: a systematic review," Archives of Dermatological Research, vol. 306, no. 7, pp. 591-599, 2014.

[11] J. Nikolovski, G. N. Stamatas, N. Kollias, and B. C. Wiegand, "Barrier function and water-holding and transport properties of infant stratum corneum are different from adult and continue to develop through the first year of life," Journal of
Investigative Dermatology, vol. 128, no. 7, pp. 1728-1736, 2008.

[12] M. O. Visscher, R. Adam, S. Brink, and M. Odio, "Newborn infant skin: physiology, development, and care," Clinics in Dermatology, vol. 33, no. 3, pp. 271-280, 2015.

[13] T. Oranges, V. Dini, and M. Romanelli, "Skin physiology of the neonate and infant: clinical implications," Advances in Wound Care, vol. 4, no. 10, pp. 587-595, 2015.

[14] B. Hughes-Formella, O. Wunderlich, R. Williams et al., "Comparison of skin structural and functional parameters in well-nourished and moderately undernourished infants," Skin Pharmacology and Physiology, vol. 32, no. 4, pp. 212-223, 2019.

[15] J. V. C. Theunis, P. Bianchi, C. C. BacqueyA et al., "Comparison of two skincare regimens in healthy newborns during the first six weeks of life," Clinical Dermatology, vol. 5, no. 3-4, pp. 99-106, 2017.

[16] J. W. Fluhr and R. Darlenski, "Skin surface $\mathrm{pH}$ in newborns: origin and consequences," $p H$ of the Skin: Issues and Challenges, vol. 54, pp. 26-32, 2018.

[17] K. Findley and E. A. Grice, "The skin microbiome: a focus on pathogens and their association with skin disease," PLoS Pathogens, vol. 10, no. 10, Article ID e1004436, 2014.

[18] K. A. Capone, S. E. Dowd, G. N. Stamatas, and J. Nikolovski, "Diversity of the human skin microbiome early in life," Journal of Investigative Dermatology, vol. 131, no. 10, pp. 2026-2032, 2011.

[19] M. G. Dominguez-Bello, E. K. Costello, M. Contreras et al., "Delivery mode shapes the acquisition and structure of the initial microbiota across multiple body habitats in newborns," Proceedings of the National Academy of Sciences, vol. 107, no. 26, pp. 11971-11975, 2010.

[20] E. K. Costello, E. M. Carlisle, E. M. Bik, M. J. Morowitz, and D. A. Relman, "Microbiome assembly across multiple body sites in low-birthweight infants," $m B i o$, vol. 4, no. 6, 13 pages, Article ID e00782, 2013.

[21] N. E. Younge, F. Araújo-Pérez, D. Brandon, and P. C. Seed, "Early-life skin microbiota in hospitalized preterm and fullterm infants," Microbiome, vol. 6, no. 1, p. 98, 2018.

[22] T. Zhu, X. Liu, F.-Q. Kong et al., "Age and mothers: potent influences of children's skin microbiota," Journal of Investigative Dermatology, vol. 139, no. 12, pp. 2497-2505, 2019.

[23] J. Lehtimäki, A. Karkman, T. Laatikainen et al., "Patterns in the skin microbiota differ in children and teenagers between rural and urban environments," Scientific Reports, vol. 7, Article ID 45651, 2017.

[24] M. B. Manus, S. Kuthyar, A. G. Perroni-Marañón, A. Núñezde la Mora, and K. R. Amato, "InfantSkin bacterial communities vary by skin site and infant age across populationsin Mexico and the United States," mSystems, vol. 5, no. 6, Article ID e00834-20, 2020.

[25] J. Kottner, L. Ludriksone, N. Garcia Bartels, and U. BlumePeytavi, "Do repeated skin barrier measurements influence each other's results? An explorative study," Skin Pharmacology and Physiology, vol. 27, no. 2, pp. 90-96, 2014.

[26] J. L. Lévêque, "EEMCO guidance for the assessment of skin topography. The European expert group on efficacy measurement of cosmetics and other topical products," Journal of the European Academy of Dermatology and Venereology : JEADV, vol. 12, no. 2, pp. 103-114, 1999.

[27] T. Gambichler, G. Moussa, P. Regeniter et al., "Validation of optical coherence tomography in vivo using cryostat histology," Physics in Medicine and Biology, vol. 52, no. 5, pp. N75-N85, 2007. 
[28] C. Trojahn, G. Dobos, C. Richter, U. Blume-Peytavi, and J. Kottner, Journal of Biomedical Optics, vol. 20, no. 4, Article ID 045003, 2015.

[29] L. Monteiro Rodrigues and J. W. Fluhr, "EEMCO guidance for the in vivo assessment of biomechanical properties of the human skin and its annexes: revisiting instrumentation and test modes," Skin Pharmacology and Physiology, vol. 33, no. 1, pp. 44-60, 2020.

[30] M. Akdeniz, S. Gabriel, A. Lichterfeld-Kottner, U. BlumePeytavi, and J. Kottner, "Transepidermal water loss in healthy adults: a systematic review and meta-analysis update," British Journal of Dermatology, vol. 179, no. 5, pp. 1049-1055, 2018.

[31] F. Elban, E. Hahnel, U. Blume-Peytavi, and J. Kottner, "Reliability and agreement of skin barrier measurements in a geriatric care setting," J Tissue Viability, vol. 29, pp. 30085-30091, 2020.

[32] H. Lambers, S. Piessens, A. Bloem, H. Pronk, and P. Finkel, "Natural skin surface $\mathrm{pH}$ is on average below 5, which is beneficial for its resident flora," International Journal of Cosmetic Science, vol. 28, no. 5, pp. 359-370, 2006.

[33] M.-H. Schmid-Wendtner and H. C. Korting, "The pH of the skin surface and its impact on the barrier function," Skin Pharmacology and Physiology, vol. 19, no. 6, pp. 296-302, 2006.

[34] E. Berardesca, "EEMCO guidance for the assessment of stratum corneum hydration: electrical methods," Skin Research and Technology, vol. 3, no. 2, pp. 126-132, 1997.

[35] P. McInnes and M. Cutting, Manual of Procedures for Humancomment Microbiome ProjectCore Microbiome Sampling Protocol AHMP Protocol \# 07-001Version Number: 12.0, 2010, https://www.ncbi.nlm.nih.gov/projects/gap/cgi-bin/ GetPdf.cgi?id=phd003190.1.

[36] I. Lozano, J. B. Saunier, S. Panhard, and G. Loussouarn, "The diversity of the human hair colour assessed by visual scales and instrumental measurements. A worldwide survey," International Journal of Cosmetic Science, vol. 39, no. 1, pp. 101-107, 2017.

[37] J. W. R. Twisk, Applied Longitudinal Data Analysis for Epidemiology: A Practical Guide, Cambridge University Press, Cambridge, UK, 2nd edition, 2013.

[38] E. von Elm, D. G. Altman, M. Egger, S. J. Pocock, P. C. Gøtzsche, and J. P. Vandenbroucke, "The strengthening the reporting of observational studies in epidemiology (STROBE) statement: guidelines for reporting observational studies," Journal of Clinical Epidemiology, vol. 61, no. 4, pp. 344-349, 2008.

[39] J. P. Vandenbroucke, E. von Elm, D. G. Altman et al., "Strengthening the reporting of observational studies in Epidemiology (STROBE): explanation and elaboration," PLoS Medicine, vol. 4, no. 10, Article ID e297, 2007. 